\title{
A Reflectivity Guided Elastic Full Waveform Inversion
}

\author{
Ehsan JAMALI HONDORI ${ }^{1}$, Hitoshi MIKADA ${ }^{2}$, and Eiichi ASAKAWA ${ }^{1}$ \\ ${ }^{1}$ Dept. of Innovative Technology Development, JGI Inc. \\ ${ }^{2}$ Dept. of Civil and Earth Res. Eng., Kyoto University
}

\begin{abstract}
Full waveform inversion (FWI) produces subsurface images by minimizing the misfit between observed data and calculated model using iterative local optimization algorithms like conjugate gradient method. This approach requires a starting model which should appear in the neighborhood of the global solution of the FWI problem to ensure that the modeled waveforms are less than half a period away from the recorded data. Usually, reflection traveltime tomography is used to create a long-wavelength background velocity model for starting FWI iterations. In this paper we suggest an alternative method to develop the starting model for FWI by using a reflectivity inversion technique. A depth section of migrated data is used to extract the reflection coefficients and impedance section, then the impedance section is converted to velocity model by considering a known density model. The reflectivity inversion can detect subsurface geological structures very well and on the other hand, an approximate known density model is a fair assumption for FWI and does not dramatically affect the long-wavelength model. We applied our method on a part of Marmousi2 model in order to develop P and S wave velocity models via elastic full waveform inversion in the frequency domain.
\end{abstract}

\section{INTRODUCTION}

Any earth model consists of short-wavelength and long-wavelength components which could be imaged by different methods. Traveltime tomography is the common technique to build a long-wavelength subsurface model and on the other hand, full waveform inversion is a robust method to develop more detailed short-wavelength images. However, nonlinearity of the FWI problem which is induced by wave propagation over a long distance and the increased number of unknowns makes successful application of FWI difficult. The method which is based on minimization of the misfit between observed data and calculated model via an iterative local optimization process requires good starting model in order to converge to the correct solution. This long-wavelength initial velocity model is insensitive to short offset data and long offset acquisitions are necessaryhe short offset dataset whereart offset data and long offset acquisitions are inevitable. -wavelength background velocity mo for recovering the smooth background velocity models, or a processing scheme should provide the initial velocity model for FWI. In this paper we suggest a reflectivity inversion process which is applied on the seismic depth section prior to FWI in order to provide a long-wavelength starting velocity model. This method first extracts the reflection coefficients from depth section by using a global optimization algorithm $^{1)}$ then an acoustic impedance section is built from the extracted reflection coefficients. Finally, by using a known density model a $\mathrm{P}$ wave velocity model is calculated which can be used as initial model for full waveform inversion. Since we deal with elastic problem, an initial S wave velocity model is also calculated using a constant Poisson's ratio.

\section{FULL WAVEFORM INVERSION}

Full waveform inversion theory has been well illustrated in literature ${ }^{2,3)}$. Misfit function is defined as the least squares norm of residuals, i.e. difference between recorded and calculated data, as

$$
E(p)=\frac{1}{2} \sum_{j=1}^{N_{s}} \Delta \mathbf{d}_{j}^{t} \Delta \mathbf{d}_{j}^{*}
$$

where, $\Delta \mathbf{d}$ is the residual wavefield and superscripts $t$ and $*$ denote transpose and complex conjugate, respectively. Summation is over source numbers and $p$ is the model parameter. Gradient of the misfit function at iteration $k$ is efficiently calculated by adjoint-state method as

$$
\mathrm{g}_{k, p}=\sum_{j=1}^{N_{s}} \mathbf{u}_{j}^{t} \frac{\partial \mathbf{A}^{t}}{\partial p} \mathbf{A}^{-1} \Delta \mathbf{d}_{j}^{*}
$$

where $\mathbf{u}$ is the modeled vector of horizontal and 
vertical displacements and $\mathbf{A}$ is the impedance matrix, i.e. the forward modeling operator in frequency domain. Preconditioned conjugate gradient method ${ }^{4)}$ is used to update the model parameter iteratively. We use diagonal approximate Hessian matrix ${ }^{5)}$ for preconditioning the gradient vector. The model parameter is updated as below

$$
\mathrm{p}_{k+1}=\mathrm{p}_{k}+\alpha \mathbf{H}^{-1} g_{k}
$$

step length $\alpha$ is calculated by a line search along the gradient direction using a parabola fitting method. Since FWI is a nonlinear and ill-posed problem there are numerous models which may equally well match the observed data. These kind of unreal solutions appear as local minimums of the misfit function and iterative methods such as gradient based algorithms are very likely to get trapped in these local minimum solutions. Therefore, in order to obtain valid results FWI iterations should start from an initial model that produces simulated waveforms which are kinematically less than half a period away from recorded data. In next section we will develop a long-wavelength velocity model by using a reflectivity inversion approach.

\section{REFLECTIVITY INVERSION}

Assuming an available depth section of seismic data, which could be the result of depth conversion of time migrated section, we define a separate inverse problem which extracts the reflection coefficients of the depth section ${ }^{1)}$. Based on the convolutional model, any seismic trace is composed of source wavelet and a reflectivity series of spikes whose amplitudes are reflection coefficients. In matrix form, the wavelet is transformed to convolution operator as below

$$
x=\mathbf{W} e+n
$$

where $x, \mathbf{W}, e$, and $n$ represent seismic trace, convolution operator, reflectivity series, and ambient noise, respectively. Although the equations are usuallyquations are written in time domainator, reflectivity series, and noise when convolved with seismic wavelet reproduce the written in time domain it is possible to apply the convolution process in any discrete domain and the mathematics are identical. In our reflectivity inversion process we search for the best set of spikes which when convolved with seismic signature reproduces the input trace with minimum misfit error. A global optimization approach is used to detect spikes locations in the reflectivity series of the stacked seismic traces by means of adaptive simulated annealing (ASA). In fact, the least squares norm of the convolutional model is minimized by a random search over spikes model space subject to

$$
F=\|x-\mathbf{W} e\|^{2}
$$

A random search sampler scans the model space and by comparing misfit value $F$, develops the best spikes set for each reflectivity trace. By inverting for all the traces in seismic section, a reflectivity section will be obtained. This reflectivity section is then converted to acoustic impedance section and by using a known density model we achieve a $\mathrm{P}$ wave velocity model. Although Jamali Hondori et al. ${ }^{1)}$ used Gardner's equation ${ }^{6}$ to relate $\mathrm{P}$ wave velocity and density we prefer using a known density model which is a fair assumption for the full waveform inversion problem. The smoothed velocity models are then used as initial models for FWI.

\section{SYNTHETIC EXAMPLE}

We selected a part of Marmousi2 model for checking our reflectivity inversion and full waveform inversion methods. The maximum size of the model in horizontal and vertical direction is $3750 \mathrm{~m}$ and $1500 \mathrm{~m}$, respectively and the model has been discretized using finite difference grids of 7.5 m. A Ricker wavelet with dominant frequency of 10 $\mathrm{Hz}$ is use with dominant frequency of $10 \mathrm{~Hz}$ re, is a suitable test $\mathrm{h}$ resemble a seismic ,22222222222222222222222222222222222 $2222222 \mathrm{~d}$ as seismic source. Figure 1(a) shows the seismic depth section which has been used for the reflectivity inversion and the resulting reflectivity section is illustrated in Figure 1(b). Adaptive simulated annealing could detect almost all of the reflection events and a reliable reflectivity model is obtained. This reflectivity section is converted to acoustic impedance section by considering the impedance of the first layer to be known. The acoustic impedance in Figure 1(c) is then converted to $\mathrm{P}$ wave velocity model using a constant density of $2000 \mathrm{~kg} / \mathrm{m}^{3}$ for the whole model. In order to use the $\mathrm{P}$ wave velocity model as the initial model for full waveform inversion we apply two different smoothing filters on velocity model. The model in Figure 1(d) is filtered using a window size of $75 \mathrm{~m}$ (initial model 1) and the second model in Figure 1(e) is filtered using a window size of $300 \mathrm{~m}$ (initial model 2). Since we deal with elastic problem, an initial $\mathrm{S}$ wave velocity model is also calculated using a constant Poisson's ratio. 
A surface reflection seismic experiment with shot interval and receiver interval of, respectively, $15 \mathrm{~m}$ and $7.5 \mathrm{~m}$ is simulated and 7 discrete frequencies are used in three different frequency groups from lower to higher values for all FWI tests. The true models of $\mathrm{P}$ and $\mathrm{S}$ wave velocities are shown in Figure 2. Before performing FWI using initial models from reflectivity inversion we used a 1D velocity model of Figure 3, which is the result of linear interpolation between minimum and maximum velocities in the true model, to show the importance of long-wavelength component of the subsurface for successful application of FWI. This 1D initial velocity model does not include any information about long-wavelength component of the model and consequently is not able to recover acceptable image of the structure, see Figure 3.
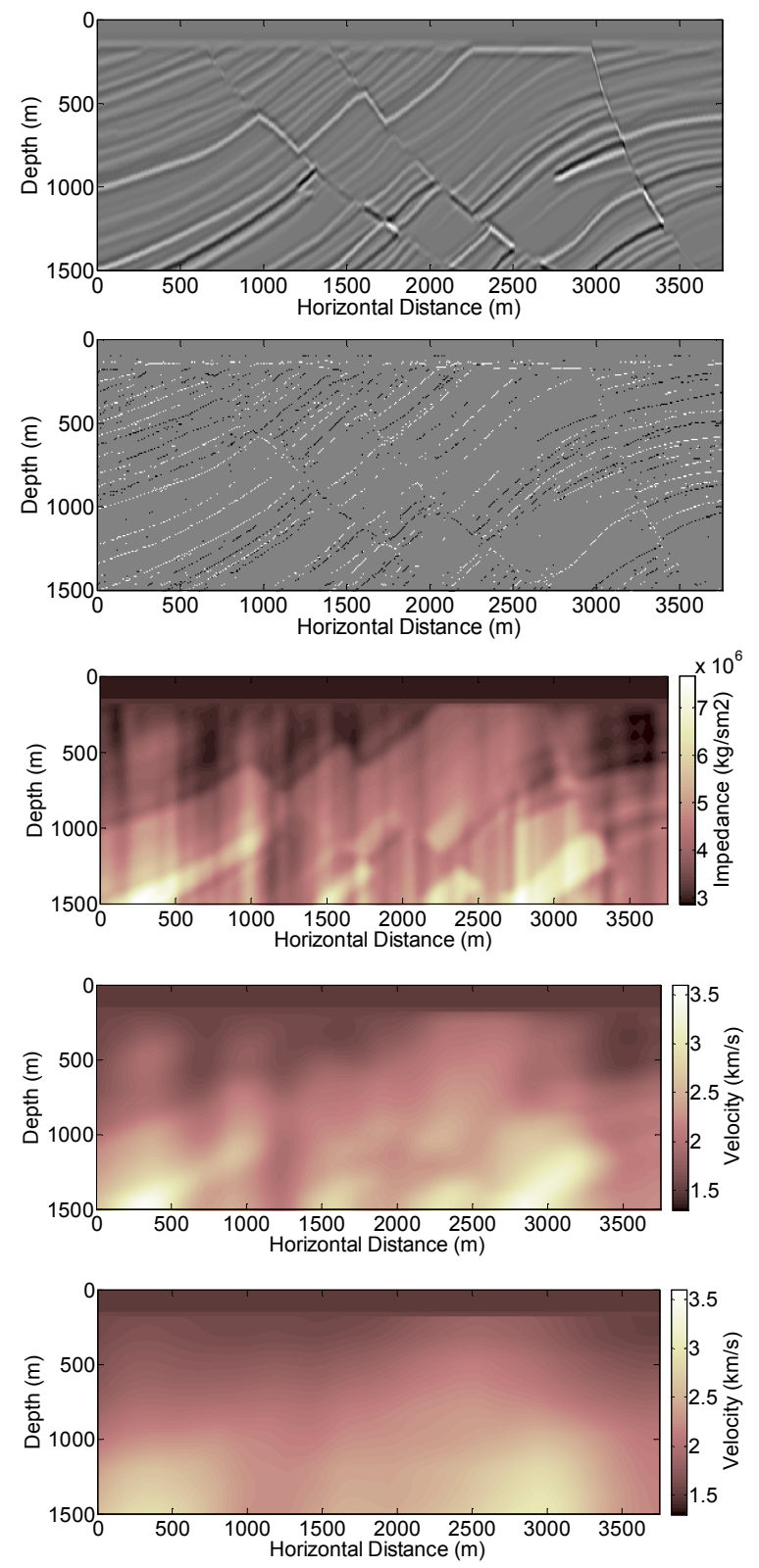

Figure 1 Top to bottom: (a) seismic depth section, (b) resulting reflectivity model, (c) acoustic impedance section, (d) smoothed velocity section with window size of 75 m (model 1), and (e) smoothed velocity section with window size of $300 \mathrm{~m}$ (model 2)

Then we used the initial models of Figure 1 to perform full waveform inversion. The FWI results are shown in Figure 4 and Figure 5. According to the results, initial model 2 which has stronger smoothing performs better compared to the initial model 1 which has weaker smoothing on it. A comparison on misfit reduction over iterations is shown in Figure 6. A vertical profile of the velocities resulting from two initial models reveals that weaker smoothing of model 1 keeps more footprint of reflectivity inversion in the starting model and probably because of a gap between inverted impedance and true impedance values, see Figure 7, this initial model could not create FWI models as well as what model 2 did. Also, initial model 2 does not include very big local effects and shows a good long-wavelength velocity model. Figure 8 shows vertical displacements simulated using true velocity models and FWI results from initial model 2. These waveforms are created by frequency domain modeling and inverse Fourier transform to produce time domain shot gathers.
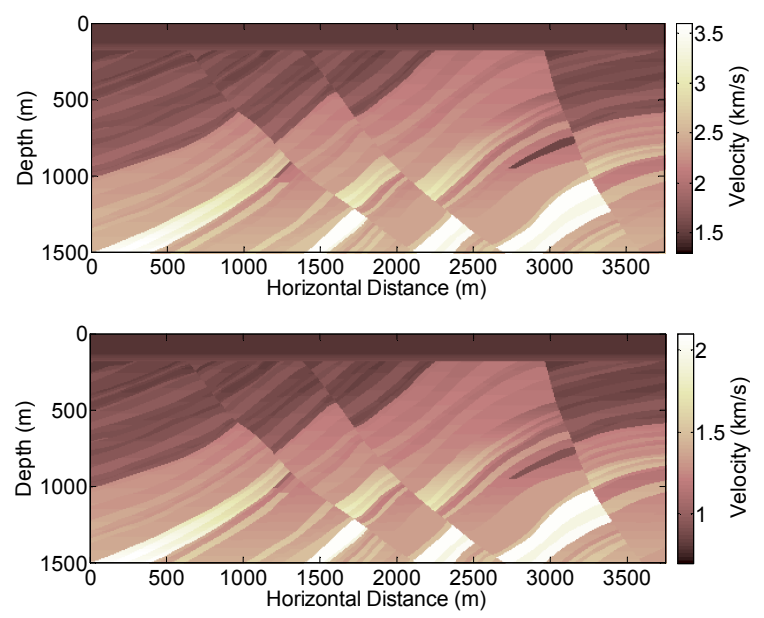

Figure $2 \mathrm{P}$ (top) and S (bottom) true velocity models.

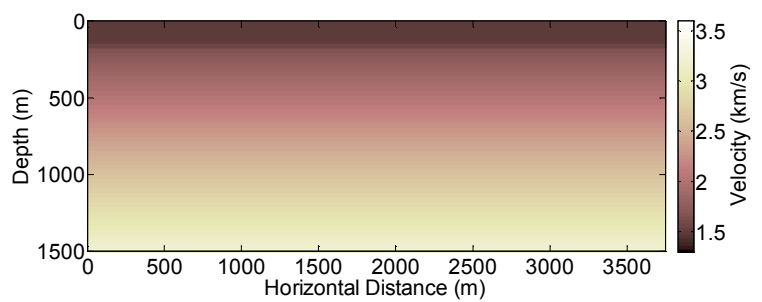




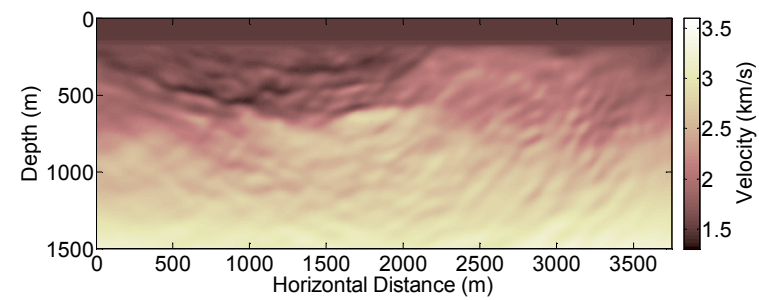

Figure 3 1D Initial $P$ wave velocity (top) and FWI result (bottom) showing importance of long-wavelength component.is not able to recover acceptable image of the structures.
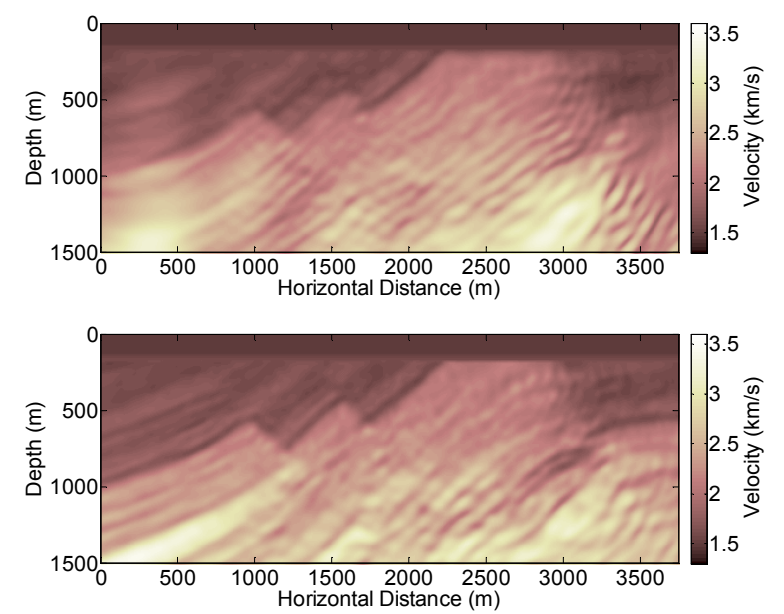

Figure $4 \mathrm{P}$ wave velocity models resulting from FWI by using initial model 1 (top) and initial model 2 (bottom)
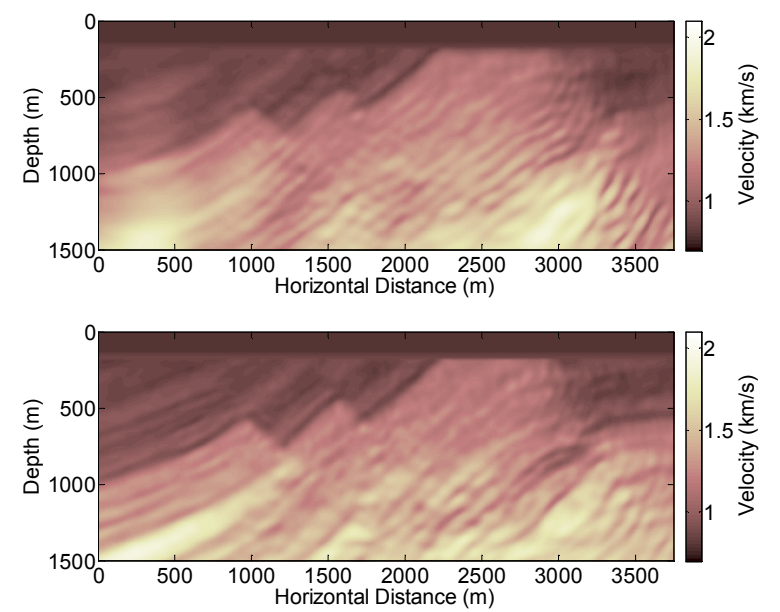

Figure $5 \mathrm{~S}$ wave velocity models resulting from FWI by using initial model 1 (top) and initial model 2 (bottom)

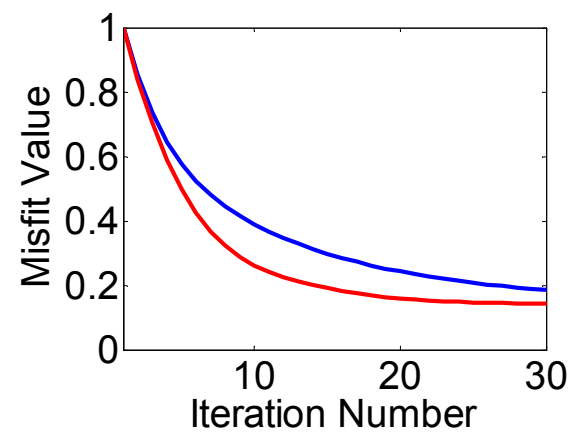

Figure 6 Misfit function of FWI results using initial model 1 (blue) and initial model 2 (red).

\section{CONCLUSIONS}

In order to converge to a satisfactory solution, FWI requires an initial model which represents long-wavelength component of the subsurface parameters. We suggested developing such an initial background velocity model by a reflectivity inversion process. Assuming an available depth seismic section and a known density model we could develop initial velocity models for FWI. Two different smoothing functions show that the initial
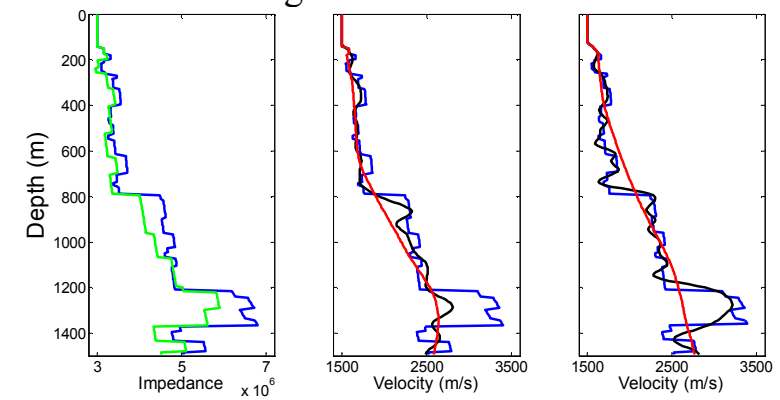

Figure 7 left: true impedance (blue), inverted impedance using reflectivity inversion (green); middle: FWI using the initial model 1 and right: FWI using initial model 2 (blue: true model, red: initial model, black: FWI result).
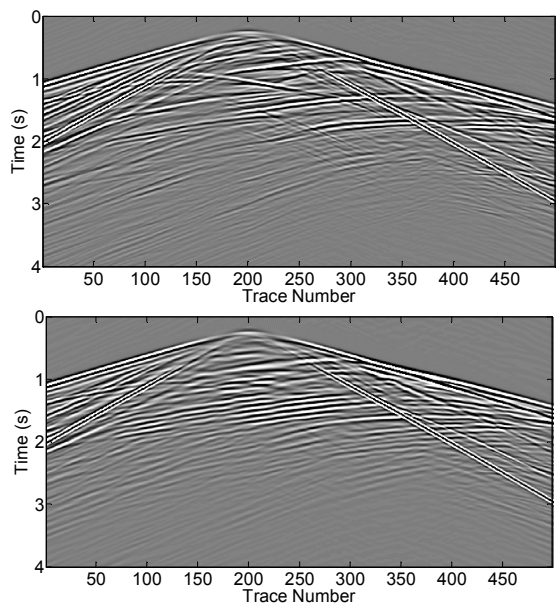

Figure 8 Top: observed vertical displacements, bottom: modeled vertical displacements after FWI using initial model 2.

model with stronger smoothing could achieve better FWI results.

\section{REFERENCES}

1) Jamali Hondori, E., Mikada, H., Goto, T. N., and Takekawa, J., 2013, A random layer-stripping method for seismic reflectivity inversion, Exploration Geophysics, 2013, 44, 70-76

2) Pratt, R. G., C. Shin, and G. J. Hicks, 1998, Gauss-Newton and full Newton methods in frequency-space seismic waveform inversion, Geophysical Journal International, 133, 341-362.

3) Virieux , J., and Operto, S., 2009, An overview of full-waveform inversion in exploration 
geophysics;, Geophysics, 74, WCC1-WCC26.

4) Vigh, D., and E. W. Starr, 2008a, 3D plane-wave full-waveform inversion, Geophysics, 73, VE135-VE144.

5) Shin, C., S. Jang, and D. J. Min, 2001, Improved amplitude preservation for prestack depth migration by inverse scattering theory, Geophysical Prospecting, 49, 592-606

6) Gardner, G. H. F., Gardner, L. W., and Gregory, A. R., 1974, Formation velocity and density: the diagnostic basics for stratigraphic traps: Geophysics, 39, 770-780 